\title{
On a slender dry patch in a liquid film draining under gravity down an inclined plane
}

\author{
S. K. WILSON ${ }^{1}$, B. R. DUFFY ${ }^{1}$ and S. H. DAVIS \\ ${ }^{1}$ Department of Mathematics, University of Strathclyde, \\ Livingstone Tower, 26 Richmond Street, Glasgow G1 1XH, UK \\ (email: s.k.wilson@strath.ac.uk) \\ ${ }^{2}$ Department of Engineering Sciences and Applied Mathematics, \\ Robert R. McCormick School of Engineering and Applied Science, \\ Northwestern University, 2145 Sheridan Road, Evanston, IL 60208, USA
}

(Received 9 October 1998; revised 21 February 2000)

\begin{abstract}
In this paper two similarity solutions describing a steady, slender, symmetric dry patch in an infinitely wide liquid film draining under gravity down an inclined plane are obtained. The first solution, which predicts that the dry patch has a parabolic shape and that the transverse profile of the free surface always has a monotonically increasing shape, is appropriate for weak surface-tension effects and far from the apex of the dry patch. The second solution, which predicts that the dry patch has a quartic shape and that the transverse profile of the free surface has a capillary ridge near the contact line and decays in an oscillatory manner far from it, is appropriate for strong surface-tension effects (in particular, when the plane is nearly vertical) and near (but not too close) to the apex of the dry patch. With the average volume flux per unit width (or equivalently with the uniform height of the layer far from the dry patch) prescribed, both solutions contain a free parameter. For each value of this parameter there is a unique solution in the first case and either no solution or a one-parameter family of solutions in the second case. The solutions capture some of the qualitative features observed in experiments.
\end{abstract}

\section{Introduction}

Understanding the formation, shape and stability of dry patches in thin liquid films on solid substrates is a fundamental fluid dynamics problem with application to numerous practical situations, including heat exchangers, distillation and absorption towers, nuclear reactors, liquid-cooled turbine blades and many coating processes.

The pioneering work on a dry patch in a liquid film draining down an inclined plane was performed by Hartley \& Murgatroyd [8], who proposed a condition for the critical maximum volume flux for a dry patch to persist based on a balance between surface tension and inertia forces at the stagnation point at its apex. Murgatroyd [17] extended Hartley \& Murgatroyd's [8] analysis to account for the presence of a gas stream above the liquid layer by incorporating the effects of surface-shear and form-drag forces. Ponter et al. [21] corrected a numerical coefficient in Hartley \& Murgatroyd's [8] analysis and found good agreement with experimental measurements of the critical flux. Hartley \& Murgatroyd's [8] approach was extended to include various thermal effects by Zuber \& 
Staub [35], McPherson [13] and Chung \& Bankoff [3]. Experiments (see, for example, the photographs of Ponter et al. [21]) show the presence of a distinctive 'capillary ridge' near the contact line which is absent from the original theory of Hartley \& Murgatroyd [8]. Wilson [30] developed a more sophisticated approximate model for the flow which incorporated the presence of this ridge. Recently, interest in the shape and stability of dry patches in a liquid film draining down an inclined plane has been renewed by the work of Podgorski et al. [19, 20], who made experimental measurements of the critical flux and the shape of dry patches at low Reynolds and capillary numbers. Finding poor agreement with Hartley \& Murgatroyd's [8] theoretical prediction of the critical flux, Podgorski et al. $[19,20]$ derived a simple approximate model for the flow similar to that of Wilson [30] based on a balance between surface-tension forces and the weight of the capillary ridge. Unlike the model of Wilson [30], their model has solutions for all values of the flux; however with the adoption of an additional assumption it yields a prediction for the critical flux which is in reasonable qualitative agreement with the experimental measurements.

Several related problems have also been investigated. The shape and stability of a hole in a static liquid film on a planar substrate was studied by Taylor \& Michael [26], and subsequently revisited by Sharma \& Ruckenstein [23], Moriarty \& Schwartz [16], Wilson \& Terrill [33], Wilson \& Duffy [31] and López, Miksis \& Bankoff [12]. The formation of rivulets and dry patches at an advancing contact line has been studied intensively both theoretically and experimentally (see, for example, the work of Huppert [11] and Troian et al. [28] and the recent review article by Oron, Davis \& Bankoff [18]) but, despite considerable advances, remains only partly understood. Other authors have used minimum-energy arguments to investigate the breakup of a uniform liquid film into rivulets, notably Hartley \& Murgatroyd [8], Bankoff [2], Mikielewicz \& Moszynski $[14,15]$ and Chung \& Bankoff [3]. The shape and stability of rivulets once they have been formed have been investigated by Towell \& Rothfeld [27], Smith [25], Allen \& Biggin [1], Wilson [30], Chung \& Bankoff [3], Davis [4], Weiland \& Davis [29], Young \& Davis [34], Schmuki \& Laso [22], Duffy \& Moffatt [5, 6] and Wilson \& Duffy [32].

The present paper is not concerned with the details of how a dry patch is formed, but instead concentrates on solutions of the governing lubrication equation that describe the possible steady dry patches that may occur. Specifically, the approach used by Smith [25] and Duffy \& Moffatt [6] to analyse a steady, slender, symmetric rivulet draining under gravity down an inclined plane from a point source is used to obtain two similarity solutions describing a steady, slender, symmetric dry patch in an infinitely wide liquid film draining under gravity down an inclined plane.

\section{Problem formulation}

Consider a thin film of viscous liquid with constant density $\rho$ and viscosity $\mu$ flowing down a planar substrate inclined at an angle $\alpha(0<\alpha \leqslant \pi / 2)$ to the horizontal. Cartesian coordinates $(x, y, z)$ with the $x$-axis down the line of greatest slope and the $z$-axis normal to the plane are adopted. Making the familiar lubrication approximation the height of the free surface $z=h(x, y, t)$ satisfies

$$
3 \mu h_{t}=\nabla \cdot\left[h^{3} \nabla\left(\rho g h \cos \alpha-\sigma \nabla^{2} h\right)\right]-\rho g \sin \alpha\left[h^{3}\right]_{x},
$$




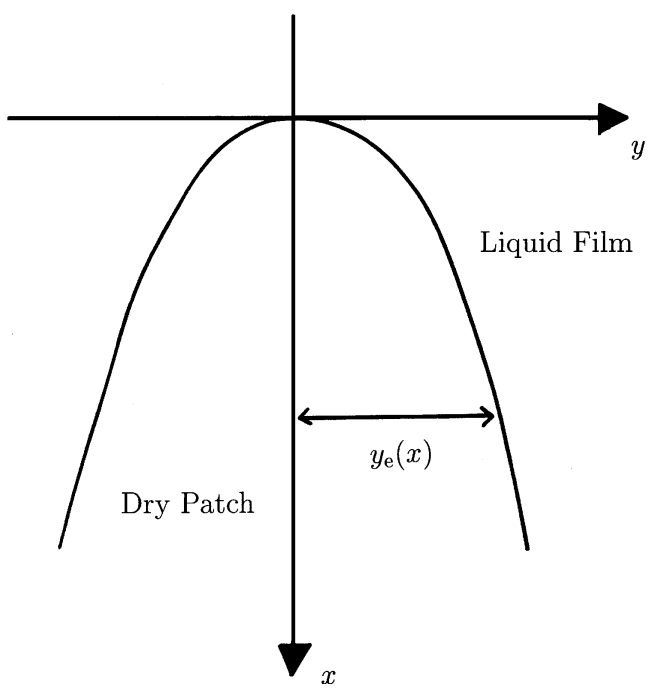

Figure 1. Geometry of the dry-patch problem.

where $t$ denotes time, $g$ denotes the magnitude of acceleration due to gravity and $\sigma$ denotes the coefficient of surface tension. Only solutions that are symmetric about $y=0$ (i.e. only solutions for which $h$ is even in $y$ ) are considered. Following the approach used by Smith [25] and Duffy \& Moffatt [6] for a slender rivulet, we seek a steady solution for a slender dry patch for which the length scale down the plane (i.e. in the $x$-direction) is much greater than in the transverse direction (i.e. in the $y$-direction), and so equation (2.1) is approximated by

$$
\left[h^{3}\left(\rho g h \cos \alpha-\sigma h_{y y}\right)_{y}\right]_{y}-\rho g \sin \alpha\left[h^{3}\right]_{x}=0 .
$$

The velocity component down the plane is $u(x, y, z)=\rho g \sin \alpha\left(2 h z-z^{2}\right) / 2 \mu$ and hence for a slender dry patch of semi-width $y_{\mathrm{e}}=y_{\mathrm{e}}(x)$ the average volume flux around the dry patch per unit width in the transverse direction down the plane, denoted by $Q(x)$, is approximately

$$
Q=\lim _{y \rightarrow \infty} y^{-1} \int_{y_{\mathrm{e}}(x)}^{y} \int_{0}^{h(x, \tilde{y})} u(x, \tilde{y}, z) \mathrm{d} z \mathrm{~d} \tilde{y}=\frac{\rho g \sin \alpha}{3 \mu} \lim _{y \rightarrow \infty} y^{-1} \int_{y_{\mathrm{e}}(x)}^{y} h(x, \tilde{y})^{3} \mathrm{~d} \tilde{y} .
$$

The geometry of the present dry-patch problem is shown in Fig. 1.

\section{Two similarity solutions}

We seek a similarity solution to equation (2.2) in the form $h=f(x) G(\eta)$, where $\eta=y / y_{\mathrm{e}}(x)$, and so, by definition, $G(1)=0$. Equation (2.2) then takes the form

$$
\rho g \cos \alpha f^{2} y_{\mathrm{e}}^{2}\left(G^{3} G^{\prime}\right)^{\prime}-\sigma f^{2}\left(G^{3} G^{\prime \prime \prime}\right)^{\prime}-3 \rho g \sin \alpha y_{\mathrm{e}}^{3} G^{2}\left(f^{\prime} G y_{\mathrm{e}}-f G^{\prime} y_{\mathrm{e}}^{\prime} \eta\right)=0,
$$

and the corresponding expression for $Q$ is

$$
Q=\frac{\rho g \sin \alpha}{3 \mu} f^{3} \lim _{\eta \rightarrow \infty} \eta^{-1} \int_{1}^{\eta} G(\tilde{\eta})^{3} \mathrm{~d} \tilde{\eta} .
$$


Inspection of equation (3.1) shows that it is not possible to obtain a similarity solution of this form satisfying the appropriate volume-flux condition when all three terms appear simultaneously. However, for weak surface-tension effects, hereafter referred to as case 1 , the second term in equation (3.1) can be neglected and the only relevant similarity solution is given (after making a suitable choice of origin in $x$ ) by $f(x)=b(c x)^{m}$ and $y_{\mathrm{e}}(x)=(c x)^{n}$, where the coefficients $b$ and $c$ and the exponents $m$ and $n$ are constants, with $m=2 n-1$. In this case $\alpha$ cannot equal $\pi / 2$ (i.e. the plane cannot be vertical) and so we may choose (without loss of generality) $b=c n \tan \alpha$, and thus equation (3.1) becomes

$$
\left[\left(G^{\prime}+\eta\right) G^{3}\right]^{\prime}-\left(7-\frac{3}{n}\right) G^{3}=0 .
$$

Similarly for strong surface-tension effects (in particular, when $\alpha$ is close to $\pi / 2$ ), hereafter referred to as case 2 , the first term in equation (3.1) can be neglected and the only relevant similarity solution is in the same form as that obtained in case 1 , but now with $m=4 n-1$. In this case we may choose (again without loss of generality) $b=\operatorname{cn} \rho g \sin \alpha / \sigma$, and thus equation (3.1) becomes

$$
\left[\left(G^{\prime \prime \prime}-\eta\right) G^{3}\right]^{\prime}+\left(13-\frac{3}{n}\right) G^{3}=0 .
$$

In both cases the remaining unknown exponent $n$ is determined by the requirement that the average volume flux per unit width around the dry patch, $Q$, be independent of $x$. This is possible only if $m=0$ and $G \sim G_{0}>0$ (a constant) as $\eta \rightarrow \infty$. Thus

$$
Q=\frac{\rho g \sin \alpha}{3 \mu}\left(b G_{0}\right)^{3}
$$

and hence

$$
m=0, \quad n=\frac{1}{2}
$$

in case 1 and

$$
m=0, \quad n=\frac{1}{4}
$$

in case 2 . Setting $n=1 / 2$ in equation (3.3) yields the governing equation in case 1 , namely

$$
\left(G^{3} G^{\prime}\right)^{\prime}+\eta\left(G^{3}\right)^{\prime}=0,
$$

while setting $n=1 / 4$ in equation (3.4) yields the governing equation in case 2 , namely

$$
\left(G^{3} G^{\prime \prime \prime}\right)^{\prime}-\eta\left(G^{3}\right)^{\prime}=0 .
$$

Regardless of the strength of surface tension, case 1 describes the leading-order balance in the limit $x \rightarrow \infty$ and case 2 the leading-order balance in the limit $x \rightarrow 0$. However, this latter statement should be treated with some caution because the requirement that the length scale down the plane is much greater than that in the transverse direction used to derive equation (2.2) means that the present solutions are expected to be valid only if $\left|h_{x}\right| \ll\left|h_{y}\right|$, i.e. where $|n y| \ll|x|$. Furthermore, the lubrication approximation used to derive equation (2.1) holds only if $\left|h_{y}\right| \ll 1$, i.e. if $\left|b G^{\prime}(\eta)\right| \ll|c x|^{n}$.

At this point it is instructive to compare the present results in case 1 and case 2 with the results for the corresponding rivulet problems obtained by Smith [25] and Duffy \& 
Moffatt [6], respectively. For a rivulet the extra condition needed to determine $n$ is the requirement that the volume flux of liquid down the plane, $Q_{R}$, given by

$$
Q_{\mathrm{R}}=2 \int_{0}^{y_{\mathrm{e}}(x)} \int_{0}^{h(x, \tilde{y})} u(x, \tilde{y}, z) \mathrm{d} z \mathrm{~d} \tilde{y}=\frac{2 \rho g \sin \alpha}{3 \mu} f^{3} y_{\mathrm{e}} \int_{0}^{1} G(\tilde{\eta})^{3} \mathrm{~d} \tilde{\eta},
$$

(rather than $Q$ ) be independent of $x$. This is possible only if $3 m+n=0$, and hence we recover the exponents obtained by Smith [25] in case 1 , namely $m=-1 / 7$ and $n=3 / 7$, and by Duffy \& Moffatt [6] in case 2, namely $m=-1 / 13$ and $n=3 / 13$. Setting $n=3 / 7$ in equation (3.3) yields $\left[\left(G^{\prime}+\eta\right) G^{3}\right]^{\prime}=0$, and imposing appropriate contact-line and symmetry conditions leads to Smith's [25] rivulet solution $G=\left(1-\eta^{2}\right) / 2$, while setting $n=3 / 13$ in equation (3.4) yields

$$
\left[\left(G^{\prime \prime \prime}-\eta\right) G^{3}\right]^{\prime}=0,
$$

and imposing appropriate contact-line and symmetry conditions leads to the oneparameter family of rivulet solutions equivalent to those obtained by Duffy \& Moffatt [6], namely

$$
G=\left(\eta^{2}-1\right)\left(\frac{\eta^{2}}{24}-\lambda\right),
$$

where $\lambda \geqslant 1 / 24$ is a free parameter. Note that Duffy $\&$ Moffatt [6] define their versions of the constants $b$ and $\lambda$ slightly differently.

For the present dry-patch problem the relevant solutions to equations (3.8) and (3.9) must satisfy the boundary condition

$$
G(1)=0
$$

and the far-field condition

$$
\lim _{\eta \rightarrow \infty} G(\eta)=G_{0} .
$$

Since equation (3.8) is of second order these two conditions may be sufficient to determine the solution in case 1. However, equation (3.9) is of fourth order and, since it is not clear what additional conditions to impose on the solution, all the possible solutions of the equation satisfying these two conditions will be described in case 2. Note that Duffy \& Moffatt [6] encountered a similar difficulty in their analysis of the corresponding rivulet problem. They imposed one contact-line and two symmetry conditions on the solution to equation (3.11), and hence obtained the one-parameter family of solutions in equation (3.12).

Unfortunately, the appropriate exact solutions of the governing equations (3.8) and (3.9) are not available. However, considerable analytical progress can still be made and the equations can be solved numerically. (There are, of course, also solutions of the governing equations that do not satisfy the boundary conditions (3.13) and (3.14), for example the exact solutions $G=-3 \eta^{2} / 7$ (case 1) and $G=\eta^{4} / 26$ (case 2), but these are not relevant to the present dry-patch problem.) 


\section{Solution in the case of weak surface tension (Case 1)}

In the case of weak surface tension (case 1), $G$ satisfies equation (3.8) subject to the boundary conditions (3.13) and (3.14), namely

$$
\begin{gathered}
\left(G^{3} G^{\prime}\right)^{\prime}+\eta\left(G^{3}\right)^{\prime}=0, \\
G(1)=0, \quad \lim _{\eta \rightarrow \infty} G(\eta)=G_{0} .
\end{gathered}
$$

As we shall describe below, this system was found numerically to have a unique solution for all $G_{0}>0$.

\subsection{Behaviour as $\eta \downarrow 1$}

Seeking a local solution of equation (3.8) near the contact line in the form $G \sim a(\eta-1)^{r}$ reveals that the local behaviour of $G$ as $\eta \downarrow 1$ is given either by

$$
G=a(\eta-1)^{\frac{1}{4}}-\frac{4}{7}(\eta-1)+\frac{48}{245 a}(\eta-1)^{\frac{7}{4}}+o(\eta-1)^{\frac{7}{4}}
$$

where, as we shall see in sections 4.3 and 4.4 , the constant $a \neq 0$ is determined by the behaviour of $G$ far from the contact line, or by

$$
G=-(\eta-1)-\frac{3}{8}(\eta-1)^{2}+o(\eta-1)^{2}
$$

Equation (4.4) has $G<0$ near $\eta=1$ and so is not relevant here. Note that the behaviour described by equation (4.3) means that the lubrication approximation fails near the contact line $\eta=1$ where the solution has infinite slope.

\subsection{Behaviour as $\eta \rightarrow \infty$}

As $\eta \rightarrow \infty$, we have $G \sim G_{0}$ and so writing $G=G_{0}+G_{1}$ in equation (3.8) and linearising for small $G_{1}$ yields

$$
G_{1}^{\prime \prime}+\frac{3 \eta}{G_{0}} G_{1}^{\prime}=0
$$

which can be integrated directly to yield

$$
G_{1}=-A\left(\frac{3 \pi}{2 G_{0}}\right)^{\frac{1}{2}}\left[\operatorname{erf}\left(\left\{\frac{3}{2 G_{0}}\right\}^{\frac{1}{2}} \eta\right)-1\right],
$$

where $A$ is an undetermined real constant. In particular, equation (4.6) shows that

$$
G \sim G_{0}+A \eta^{-1} \exp \left(-\frac{3 \eta^{2}}{2 G_{0}}\right)
$$

as $\eta \rightarrow \infty$, and hence that $G$ approaches $G_{0}$ monotonically in this limit.

\subsection{The singular limit $G_{0} \rightarrow 0$}

We can investigate the singular limit $G_{0} \rightarrow 0$ by writing $G=G_{0} \hat{G}$, in which case equation (3.8) becomes

$$
G_{0}\left(\hat{G}^{3} \hat{G}^{\prime}\right)^{\prime}+\eta\left(\hat{G}^{3}\right)^{\prime}=0
$$


In the limit $G_{0} \rightarrow 0$ the leading-order version of equation (4.8) is $\hat{G}^{\prime}=0$, and so the appropriate solution is simply $\hat{G}=1$. This outer solution evidently fails near the contact line at $\eta=1$ and so in this inner region we introduce a rescaled coordinate defined by $\eta=1+G_{0} \hat{\eta}$; then (using the matching condition with the outer solution) at leading order equation (4.8) becomes

$$
\hat{G}^{\prime}=\hat{G}^{-3}-1 .
$$

Equation (4.9) can be solved exactly, and imposing the contact-line condition $\hat{G}=0$ at $\hat{\eta}=0$ yields the implicit solution

$$
\hat{\eta}=\frac{1}{\sqrt{3}}\left[\arctan \left(\frac{1+2 \hat{G}}{\sqrt{3}}\right)-\frac{\pi}{6}\right]-\hat{G}-\frac{1}{3} \log (1-\hat{G})+\frac{1}{6} \log \left(1+\hat{G}+\hat{G}^{2}\right),
$$

which is plotted in Fig. 2. Since the leading-order outer solution is a constant, the uniformly valid leading-order composite solution is simply

$$
G=G_{0} \hat{G}\left(\frac{\eta-1}{G_{0}}\right) .
$$

In particular, this solution satisfies

$$
G=\sqrt{2} G_{0}^{\frac{3}{4}}(\eta-1)^{\frac{1}{4}}-\frac{4}{7}(\eta-1)+\frac{48}{245 \sqrt{2} G_{0}^{\frac{3}{4}}}(\eta-1)^{\frac{7}{4}}+o(\eta-1)^{\frac{7}{4}}
$$

as $\eta \downarrow 1$, which coincides with the corresponding result in equation (4.3) up to the order shown provided that $a=\sqrt{2} G_{0}^{3 / 4}$. Also

$$
G \sim G_{0}-\sqrt{3} G_{0} \exp \left(-3+\frac{\pi}{2 \sqrt{3}}+\frac{3}{G_{0}}\right) \exp \left(-\frac{3 \eta}{G_{0}}\right)
$$

as $\eta \rightarrow \infty$, which differs somewhat from the corresponding result in equation (4.7), but shows that $G$ still approaches $G_{0}$ monotonically in this limit.

\subsection{Numerical solution}

The system (3.8), (3.13) and (3.14) can be solved numerically using a Runge-Kutta method.

The numerical calculation can be started from $\eta=1+\delta$ for sufficiently small $\delta \ll 1$ by using equation (4.3) to give the approximate initial conditions

$$
\left.\begin{array}{rl}
G(1+\delta) & =a \delta^{\frac{1}{4}}-\frac{4}{7} \delta+\frac{48}{245 a} \delta^{\frac{7}{4}} \\
G^{\prime}(1+\delta) & =\frac{a}{4} \delta^{-\frac{3}{4}}-\frac{4}{7}+\frac{12}{35 a} \delta^{\frac{3}{4}}
\end{array}\right\}
$$

With these initial conditions the equation can be integrated forwards in $\eta$ until the limiting value of $G=G_{0}$ is approached sufficiently closely for a range of values of $a$, and thus the relationship between $a$ and $G_{0}$ determined.

Alternatively, the numerical calculation can be started from $\eta=X$ for sufficiently large $X \gg 1$ by using equation (4.7) to give the approximate initial conditions

$$
\left.\begin{array}{rl}
G(X) & =G_{0}+A X^{-1} \exp \left(-B X^{2}\right), \\
G^{\prime}(X) & =-2 A B \exp \left(-B X^{2}\right),
\end{array}\right\}
$$




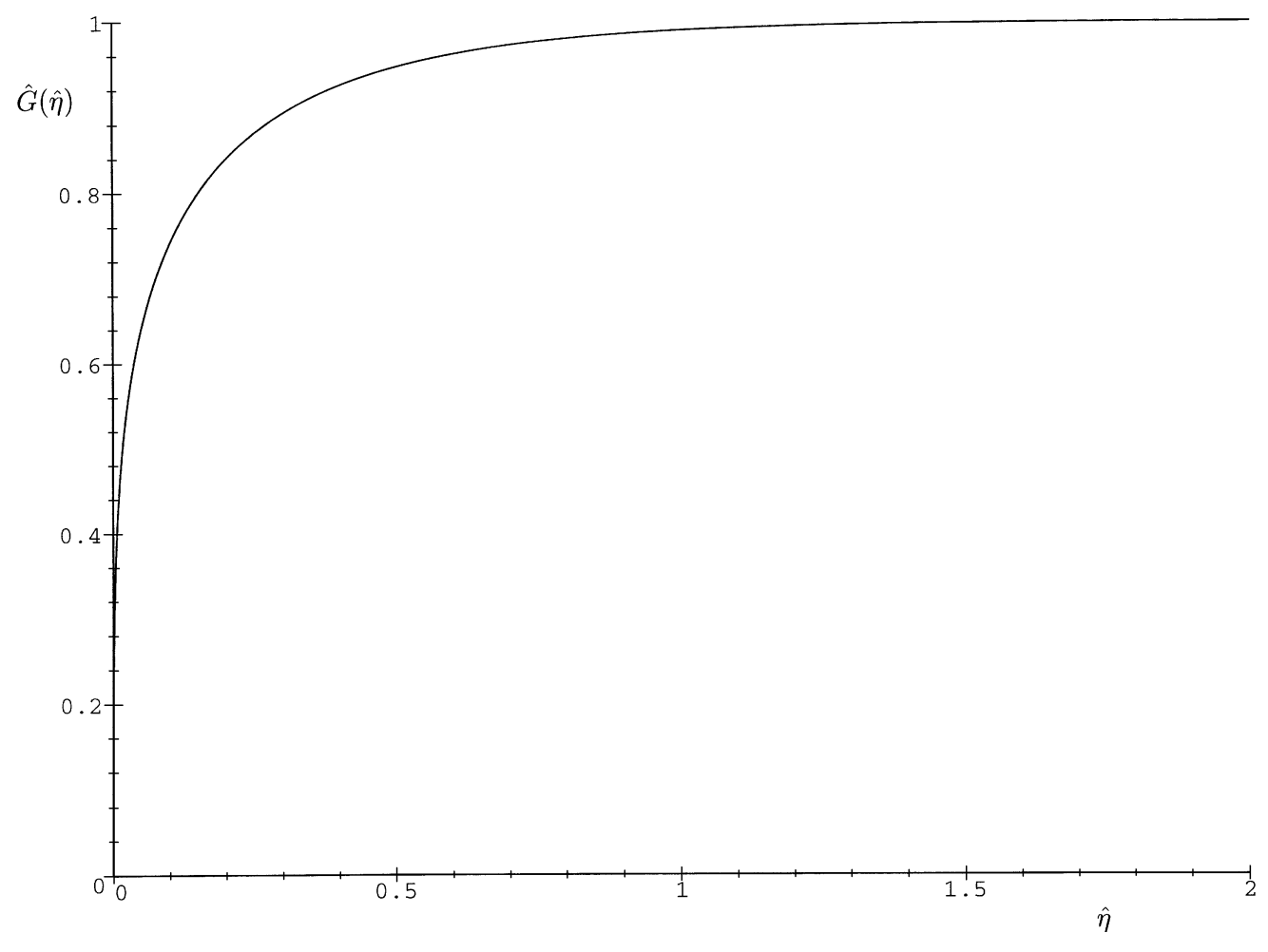

FIGURE 2. The inner solution for the free-surface profile in the limit $G_{0} \rightarrow 0, \hat{G}(\hat{\eta})$, in case 1 given by equation (4.10) plotted as a function of $\hat{\eta}$.

Table 1. Numerically calculated values of $G_{0}$ for a range of values of a in case 1

\begin{tabular}{lllcrcrcr}
\hline \hline$a$ & \multicolumn{1}{c}{$G_{0}$} & \multicolumn{1}{c}{$a$} & \multicolumn{1}{c}{$G_{0}$} & \multicolumn{1}{c}{$a$} & \multicolumn{1}{c}{$G_{0}$} & \multicolumn{1}{c}{$a$} & \multicolumn{1}{c}{$G_{0}$} \\
\hline 0.01 & 0.001357 & 0.5 & 0.2456 & 5 & 4.6514 & 40 & 56.741 \\
0.05 & 0.01159 & 0.6 & 0.3119 & 10 & 10.857 & 50 & 73.759 \\
0.1 & 0.02917 & 0.7 & 0.3815 & 15 & 17.699 & 75 & 118.56 \\
0.2 & 0.07325 & 0.8 & 0.4541 & 20 & 24.699 & 100 & 165.81 \\
0.3 & 0.1253 & 0.9 & 0.5292 & 25 & 32.555 & $10^{3}$ & 2368.7 \\
0.4 & 0.1832 & 1 & 0.6066 & 30 & 40.406 & $10^{4}$ & 33165 \\
\hline \hline
\end{tabular}

where $B=3 / 2 G_{0}$. With these initial conditions the equation can be integrated backwards in $\eta$ and the value of $A$ iterated until the contact line occurs sufficiently close to $\eta=1$ for a range of values of $G_{0}$. In principle, the value of $a$ can then be calculated and thus the relationship between $a$ and $G_{0}$ once again determined. However, in practice the difficulty in obtaining sufficiently accurate solutions for $G$ near the contact line makes precise determination of $a$ using this method problematic.

Since the first method is much easier to implement, the numerically calculated values of $G_{0}$ given in Table 1 and plotted in Fig. 3 for a range of values of $a$ were calculated this way using the MAPLE V computer algebra package. Selected values of $A$ were then 


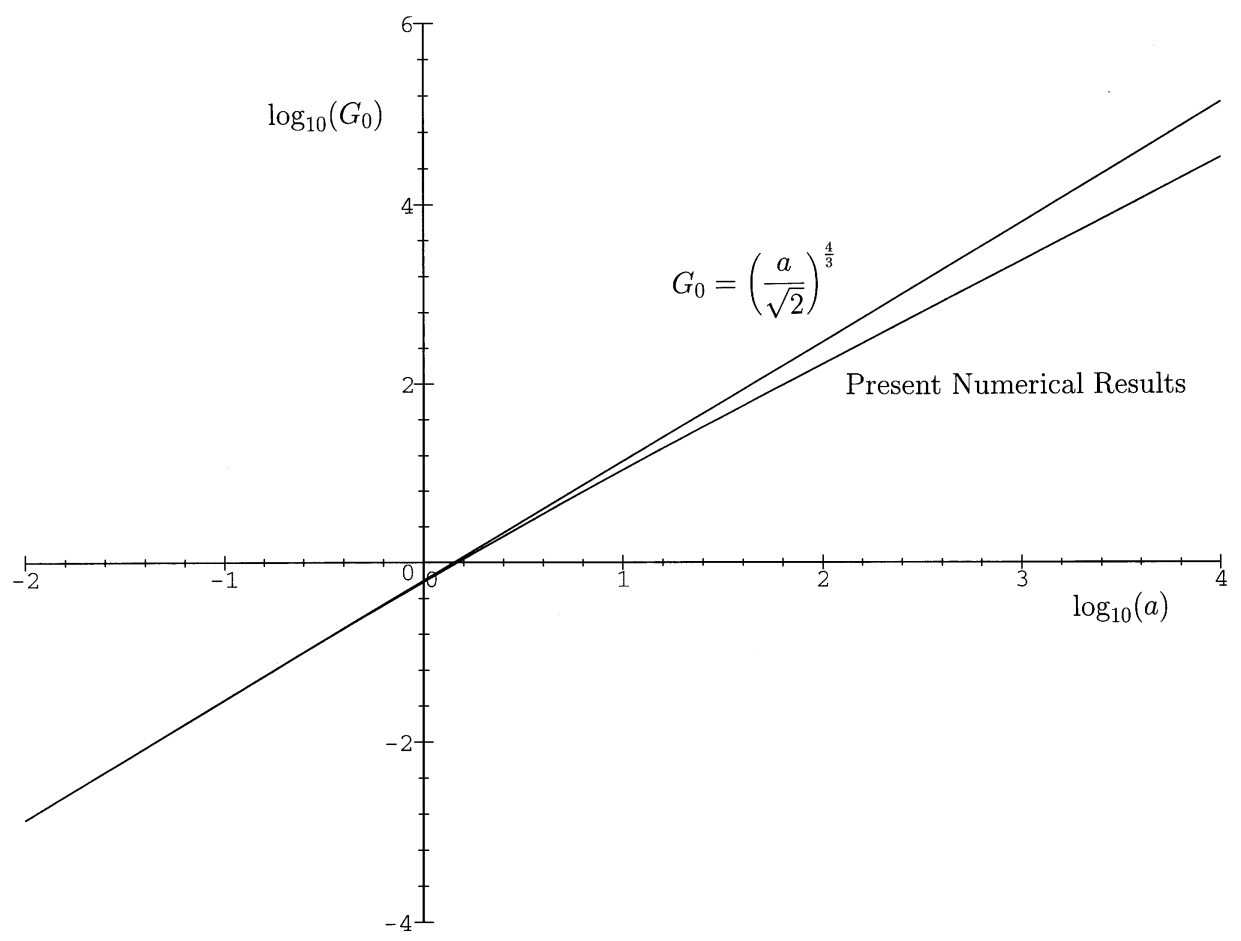

FIGURE 3. Numerically calculated values of $\log _{10}\left(G_{0}\right)$ in case 1 plotted as a function of $\log _{10}(a)$. The corresponding leading-order asymptotic value of $G_{0}$ in the limit $G_{0} \rightarrow 0$, namely $G_{0}=(a / \sqrt{2})^{4 / 3}$, is also shown.

double-checked using the second method. Figure 3 also shows that the leading-order asymptotic value of $G_{0}$ in the limit $G_{0} \rightarrow 0$ calculated in $\S 4.3$, namely $G_{0}=(a / \sqrt{2})^{4 / 3}$, is in excellent agreement with the numerical results for sufficiently small values of $G_{0}$. Typical numerically calculated free-surface profiles are plotted in Fig. 4 for a range of values of $a$. The numerical results indicate that $G_{0}$ is a monotonically increasing function of $a$, that for every value of $G_{0}$ there is a corresponding unique value of $a$, and that in each case the corresponding free surface has a simple monotonically increasing profile.

\section{Solution in the case of strong surface tension (Case 2)}

In the case of strong surface tension (case 2), $G$ satisfies equation (3.9) subject to the boundary conditions (3.13) and (3.14), namely

$$
\begin{gathered}
\left(G^{3} G^{\prime \prime \prime}\right)^{\prime}-\eta\left(G^{3}\right)^{\prime}=0, \\
G(1)=0, \quad \lim _{\eta \rightarrow \infty} G(\eta)=G_{0} .
\end{gathered}
$$

As we shall describe below, this system was found numerically to have no solution when $G_{0}$ is less than the critical value $G_{0 c} \approx 2.4$, and a one-parameter family of solutions when $G_{0} \geqslant G_{0 c}$. 


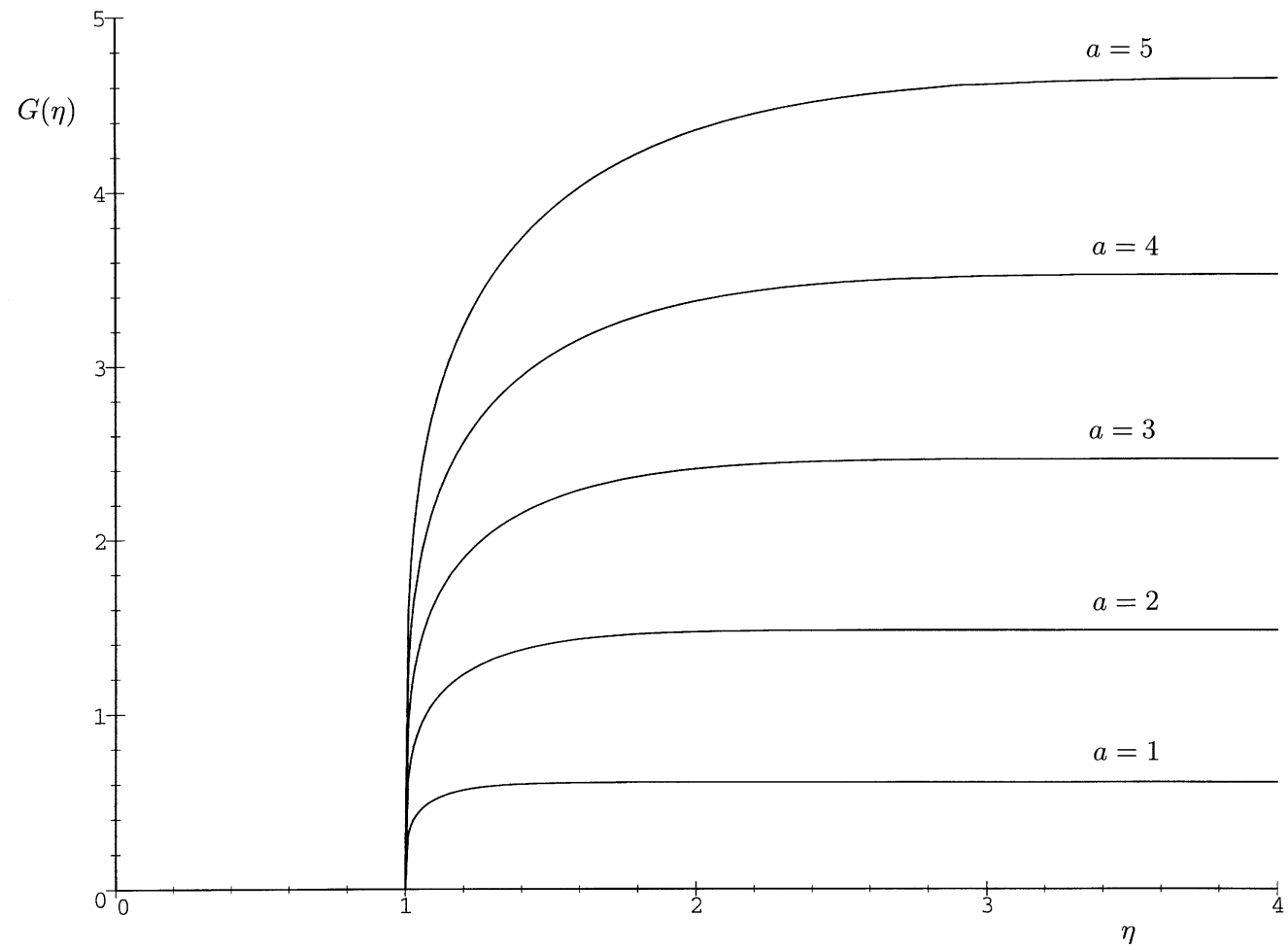

FiguRE 4. Numerically calculated free-surface profiles $G(\eta)$ in case 1 plotted as a function of $\eta$ for $a=1,2,3,4$ and 5 .

\subsection{Behaviour as $\eta \downarrow 1$}

Proceeding as in case 1 reveals that the local behaviour of $G$ as $\eta \downarrow 1$ is given either by

$$
G=a(\eta-1)^{\frac{3}{4}}+\frac{64}{429}(\eta-1)^{3}+\frac{192}{6851}(\eta-1)^{4}+o(\eta-1)^{4},
$$

where again the constant $a \neq 0$ is determined by the behaviour of $G$ far from the contact line, or by

$$
G=\frac{1}{6}(\eta-1)^{3}+\frac{3}{80}(\eta-1)^{4}+o(\eta-1)^{4} .
$$

In this case there is no immediate reason for disregarding equation (5.4) as there was for disregarding equation (4.4) in case 1 . However, as we shall see in $\S 5.4$, the present numerical results indicate that the behaviour described by equation (5.4) is not relevant here. Note that the behaviour described by equation (5.3) means that the lubrication approximation again fails near the contact line $\eta=1$ where the solution has infinite slope.

\subsection{Behaviour as $\eta \rightarrow \infty$}

As $\eta \rightarrow \infty$ we have $G \sim G_{0}$ and so writing $G=G_{0}+G_{1}$ in equation (3.9) and linearising for small $G_{1}$ yields

$$
G_{1}^{\prime \prime \prime \prime}-\frac{3 \eta}{G_{0}} G_{1}^{\prime}=0
$$


Unlike for the corresponding equation in case 1 (equation (4.5)), the exact solution of this equation is not available. However, valuable information about the behaviour of $G_{1}$ in the limit $\eta \rightarrow \infty$ can be obtained by seeking an asymptotic solution in the form $G_{1} \sim \hat{A} \eta^{p} \exp \left(-\hat{B} \eta^{q}\right)$, where the complex constants $\hat{A}$ and $\hat{B}$ (where $\operatorname{Re}(\hat{B})>0$ ) and the real exponents $p$ and $q>0$ are to be determined. Substituting this solution into equation (5.5) yields

$$
p=-\frac{2}{3}, \quad q=\frac{4}{3}, \quad \hat{B}=\frac{3}{8}\left(\frac{3}{G_{0}}\right)^{\frac{1}{3}}(1 \pm \sqrt{3} \mathrm{i}),
$$

but leaves $\hat{A}$ undetermined. Thus we have

$$
G \sim G_{0}+A \eta^{-\frac{2}{3}} \exp \left[-\frac{3}{8}\left(\frac{3}{G_{0}}\right)^{\frac{1}{3}} \eta^{\frac{4}{3}}\right] \cos \left[\frac{3 \sqrt{3}}{8}\left(\frac{3}{G_{0}}\right)^{\frac{1}{3}} \eta^{\frac{4}{3}}+\phi\right]
$$

as $\eta \rightarrow \infty$, where $A$ is an undetermined real constant and $\phi$ is an undetermined phase shift in the interval $[0, \pi)$, and so $G$ approaches $G_{0}$ in an oscillatory manner in this case. ${ }^{1}$

\subsection{The singular limit $G_{0} \rightarrow 0$}

Following the same approach as that used successfully in case 1, we investigate the singular limit $G_{0} \rightarrow 0$ by writing $G=G_{0} \hat{G}$, in which case equation (3.9) becomes

$$
G_{0}\left(\hat{G}^{3} \hat{G}^{\prime \prime \prime}\right)^{\prime}-\eta\left(\hat{G}^{3}\right)^{\prime}=0 .
$$

In the limit $G_{0} \rightarrow 0$ the leading-order version of equation (5.8) is again $\hat{G}^{\prime}=0$ with the appropriate solution $\hat{G}=1$. Once again this outer solution fails near the contact line at $\eta=1$ and so in this inner region we introduce a rescaled coordinate defined by $\eta=1+G_{0}^{1 / 3} \hat{\eta}$; then (using the matching condition with the outer solution) at leading order equation (5.8) becomes

$$
\hat{G}^{\prime \prime \prime}=1-\hat{G}^{-3} \text {. }
$$

Unlike for the corresponding equation in case 1 (equation (4.9)), we have been unable to find an exact solution to this equation that satisfies the contact-line condition $\hat{G}=0$ at $\hat{\eta}=0$. For example, seeking a solution to the equation near $\hat{\eta}=0$ in the form $\hat{G} \sim a \hat{\eta}^{r}$ requires that $r=3 / 4$ (in agreement with equation (5.3)), but also requires that $a$ be complex (specifically that $a=\left(-4^{3} / 15\right)^{1 / 4}$ ), which is impossible. The apparent failure of this procedure suggests that there may, in fact, be no solution to the problem in this limit. This tentative conclusion is confirmed by the numerical results presented below.

\subsection{Numerical solution}

As in case 1, the system (3.9), (3.13) and (3.14) can be solved numerically using a Runge-Kutta method.

Motivated by the numerical approach used successfully in case 1 , the first numerical calculations were started from $\eta=1+\delta$ for sufficiently small $\delta \ll 1$ using equations (5.3)

1 Applying the same procedure to equation (4.5) in case 1 yields $p=-1, q=2$ and $\hat{B}=3 / 2 G_{0}$ (but again leaves $\hat{A}$ undetermined) in exact agreement with equation (4.7). 
and (5.4) to give approximate initial conditions analogous to those in equation (4.14) in case 1. However, with either equation (5.3) or (5.4), this procedure yielded only solutions for $G$ that grow unboundedly as $\eta \rightarrow \infty$, and hence do not approach a limiting value as $\eta \rightarrow \infty$, as required. Therefore, to ensure the boundedness of $G$ as $\eta \rightarrow \infty$, all the numerical results presented here were instead calculated by starting from $\eta=X$ for sufficiently large $X \gg 1$, with equation (5.7) used to give the approximate initial conditions

$$
\left.\begin{array}{rl}
G(X) & =G_{0}+A \exp \left(-\hat{B}_{r} X^{\frac{4}{3}}\right) X^{-\frac{2}{3}} C \\
G^{\prime}(X) & =A \exp \left(-\hat{B}_{r} X^{\frac{4}{3}}\right)\left(-\frac{4}{3}\right) X^{-\frac{1}{3}}\left(\hat{B}_{r} C+\hat{B}_{i} S\right), \\
G^{\prime \prime}(X) & =A \exp \left(-\hat{B}_{r} X^{\frac{4}{3}}\right)\left(-\frac{4}{3}\right)^{2}\left[\left(\hat{B}_{r}^{2}-\hat{B}_{i}^{2}\right) C+2 \hat{B}_{r} \hat{B}_{i} S\right], \\
G^{\prime \prime \prime}(X) & =A \exp \left(-\hat{B}_{r} X^{\frac{4}{3}}\right)\left(-\frac{4}{3}\right)^{3} X^{\frac{1}{3}}\left[\left(\hat{B}_{r}^{2}-3 \hat{B}_{i}^{2}\right) \hat{B}_{r} C+\left(3 \hat{B}_{r}^{2}-\hat{B}_{i}^{2}\right) \hat{B}_{i} S\right],
\end{array}\right\}
$$

where $\hat{B}=\hat{B}_{r}+\mathrm{i} \hat{B}_{i}$ is defined in equation (5.6), $S=\sin \left(\hat{B}_{i} X^{\frac{4}{3}}+\phi\right)$ and $C=\cos \left(\hat{B}_{i} X^{\frac{4}{3}}+\phi\right)$. The solutions calculated in this manner do not, in general, satisfy the contact line condition (3.13). Indeed, they may not even have a contact line at all. However, in all the cases when a contact line is present the numerically calculated slope near the contact line is very large, indicating that the local behaviour is given by equation (5.3), which has infinite slope at the contact line, and not by equation (5.4), which has zero slope there. However, the difficulty in obtaining sufficiently accurate solutions for $G$ near the contact line again makes precise determination of $a$ in equation (5.3) using this method problematic and so we do not attempt to do this in this case.

Figure 5 shows numerically calculated solutions for $G$ plotted as a function of $\eta$ for several different values of $A$ in the case $\phi=0$ and $G_{0}=1$. For $A>A_{c}$, where $A_{c} \approx-2.6$, the solutions for $G$ diverge as $\eta \rightarrow-\infty$ and do not have a contact line, while for $A \leqslant A_{c}$ they have a contact line at $\eta=\eta^{*}$ which is, in general, not equal to unity. When $A=A_{c}$ then $\eta^{*} \approx 0.73$, and decreasing $A$ increases $\eta^{*}$ until a maximum value of $\eta^{*} \approx 0.80$ is reached when $A \approx-4.0$, after which $\eta^{*}$ decreases as $A$ is decreased further. Qualitatively similar behaviour occurs for other values of $\phi$. This behaviour is summarised in Fig. 6, in which we plot $G_{0}^{-1 / 4} \eta^{*}$ as a function of $G_{0}^{-7 / 6} A$ for a range of values of $\phi$ in the case $G_{0}=1$. (The reason for scaling $\eta^{*}$ and $A$ with $G_{0}$ in this way will become apparent shortly.) As Fig. 6 shows, when $\phi$ is increased from zero the curves for $\eta^{*}$ as a function of $A$ for fixed $\phi$ move downwards, and for sufficiently large values of $\phi$ satisfying $\phi>\phi_{c}$, where $\phi_{c} \approx 0.35 \pi$, the values of $\eta^{*}$ are negative for all values of $A$ and so do not appear on Fig. 6 at all. As Fig. 6 also shows, there is no combination of values of $A$ and $\phi$ for which $\eta^{*}=1$ (i.e. no solution that satisfies $G(1)=0$ ), and so we deduce that there is no solution of the kind we seek in the case $G_{0}=1$.

There are, however, solutions that satisfy $G(1)=0$ for larger values of $G_{0}$. In fact, Fig. 6 contains all the information we need about the solutions when $G_{0} \neq 1$. To understand this we write

$$
G(\eta)=G_{0} \bar{G}(\bar{\eta}), \quad \eta=G_{0}^{\frac{1}{4}} \bar{\eta}, \quad A=G_{0}^{\frac{7}{6}} \bar{A},
$$

where $\bar{G}=\bar{G}(\bar{\eta})$ is exactly the solution of the problem in the case $G_{0}=1$ described above with $\bar{A}$ in place of $A$. Thus plotting $G_{0}^{-1 / 4} \eta^{*}$ as a function of $G_{0}^{-7 / 6} A$ as we have done in Fig. 6 gives the same curves for all values of $G_{0}{ }^{2}$ Inspection of Fig. 6 shows that

${ }^{2}$ The corresponding scalings for $\eta^{*}$ and $A$ in case 1 are $G_{0}^{-1 / 2}$ and $G_{0}^{-3 / 2}$, respectively. 


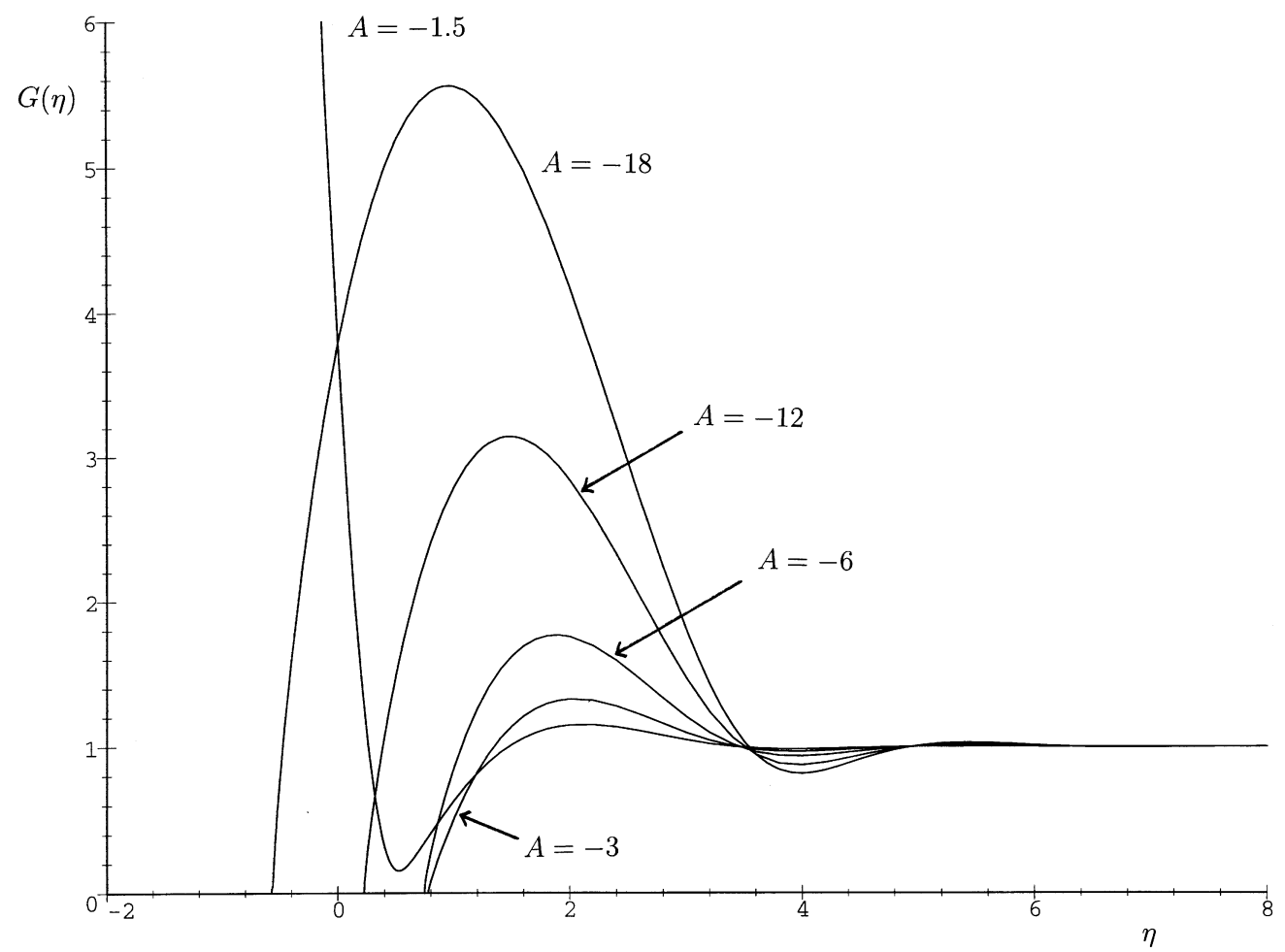

FIGURE 5. Numerically calculated free-surface profiles $G(\eta)$ in case 2 plotted as a function of $\eta$ when $\phi=0$ and $G_{0}=1$ for $A=-1.5,-3,-6,-12$ and -18 . Note that none of these profiles satisfies $G(1)=0$.

solutions with $\eta^{*}=1$ (i.e. solutions that satisfy $G(1)=0$ ) are impossible if $G_{0}<G_{0 c}$, where $G_{0 c} \approx(0.80)^{-4} \approx 2.4$, but for $G_{0} \geqslant G_{0 c}$ there are solutions for values of $\phi$ in the interval $\left[0, \phi_{\max }\right]$, where the value of $\phi_{\max }\left(\leqslant \phi_{c}\right)$ depends on $G_{0}$.

In particular, Fig. 6 shows that when $\phi=0$ there are no solutions in either of the cases $G_{0}=1$ or $G_{0}=2$, two solutions in the case $G_{0}=3$, and one solution in each of the cases $G_{0}=4$ and $G_{0}=5$. These solutions are shown in Fig. 7. Figure 6 also shows that when $G_{0}=25\left(>G_{0 c}\right)$ there is one solution with $\phi=0$ and $\phi=\pi / 16$, two solutions with $\phi=\pi / 8$ but no solutions with $\phi=3 \pi / 16$ or larger. These solutions are shown in Fig. 8. All the solutions shown in Figs. 7 and 8 have a characteristic capillary ridge near the contact line which decays in an oscillatory manner as $\eta \rightarrow \infty$, and which is qualitatively similar to those observed both experimentally and theoretically near moving contact lines in a variety of physical contexts.

\section{Conclusions}

In this paper, two similarity solutions describing a steady, slender, symmetric dry patch in an infinitely wide liquid film draining under gravity down an inclined plane were obtained. 


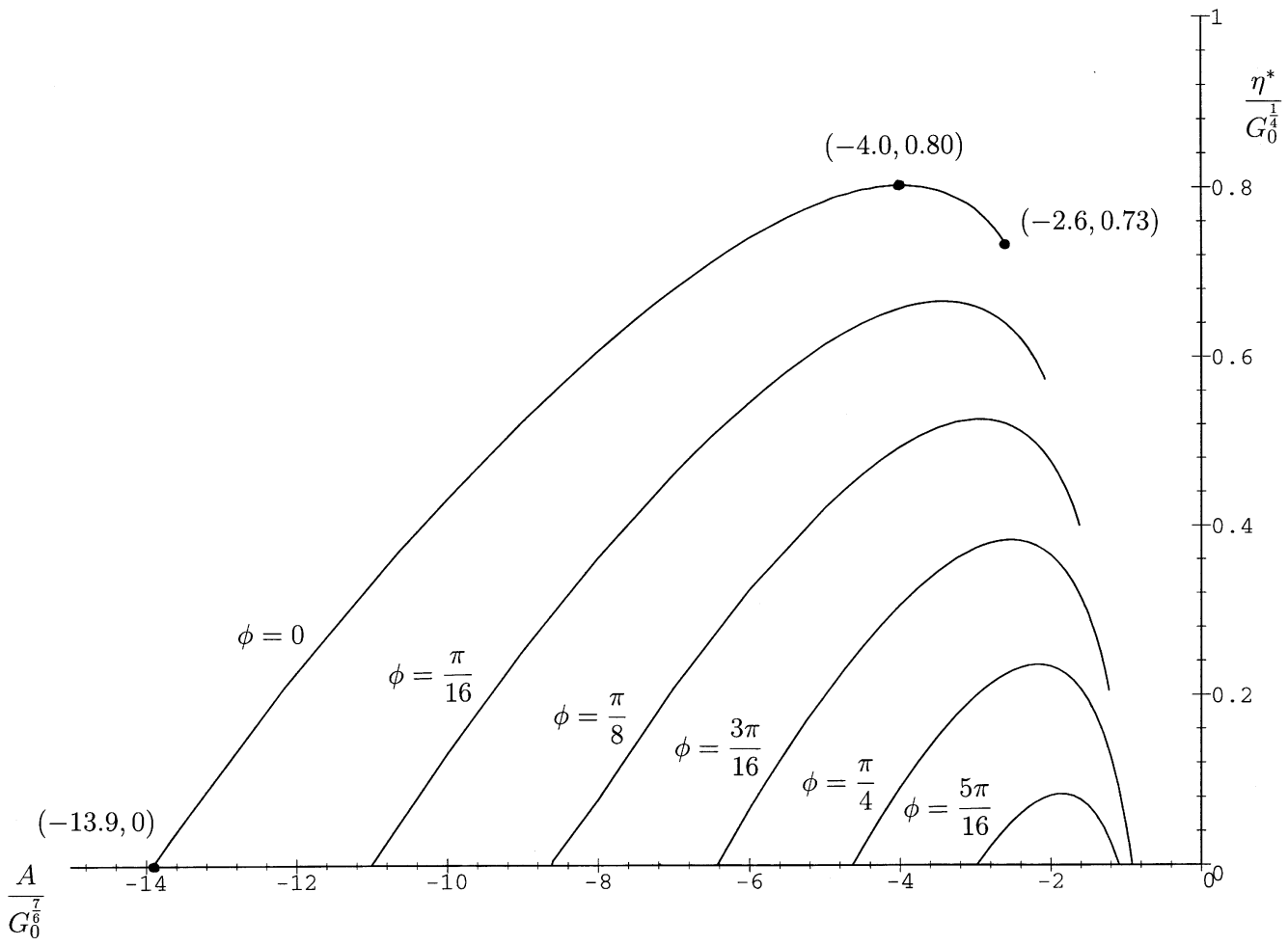

FIGURE 6. Numerically calculated values of the scaled contact-line position $G_{0}^{-1 / 4} \eta^{*}$ in case 2 plotted as a function of $G_{0}^{-7 / 6} A$ for a range of values of $\phi$.

In the case of weak surface tension (case 1), the solution takes the form

$$
h=\frac{c \tan \alpha}{2} G\left(\frac{y}{(c x)^{\frac{1}{2}}}\right), \quad Q=\frac{\rho g \sin \alpha}{3 \mu}\left(\frac{c \tan \alpha G_{0}}{2}\right)^{3},
$$

where $c>0$ and $G$ satisfies equation (3.8) subject to the boundary conditions (3.13) and (3.14). This solution, which predicts that the dry patch has the parabolic shape $y=(c x)^{\frac{1}{2}}$ and that the transverse profile of the free surface has a monotonically increasing shape, is appropriate for weak surface-tension effects and far from the apex of the dry patch. In this case there is a unique solution for $G$ for all values of $G_{0}$.

In the case of strong surface tension (case 2), the solution takes the form

$$
h=\frac{c \rho g \sin \alpha}{4 \sigma} G\left(\frac{y}{(c x)^{\frac{1}{4}}}\right), \quad Q=\frac{\rho g \sin \alpha}{3 \mu}\left(\frac{c \rho g \sin \alpha G_{0}}{4 \sigma}\right)^{3},
$$

where $c>0$ and $G$ satisfies equation (3.9) subject to the same boundary conditions (3.13) and (3.14) as in case 1. This solution, which predicts that the dry patch has the quartic shape $y=(c x)^{\frac{1}{4}}$ and that the transverse profile of the free surface has a capillary ridge near the contact line and decays in an oscillatory manner far from it, is appropriate for strong surface-tension effects (in particular, when the plane is nearly vertical) and near (but not too close) to the apex of the dry patch. In this case, there is no solution when 


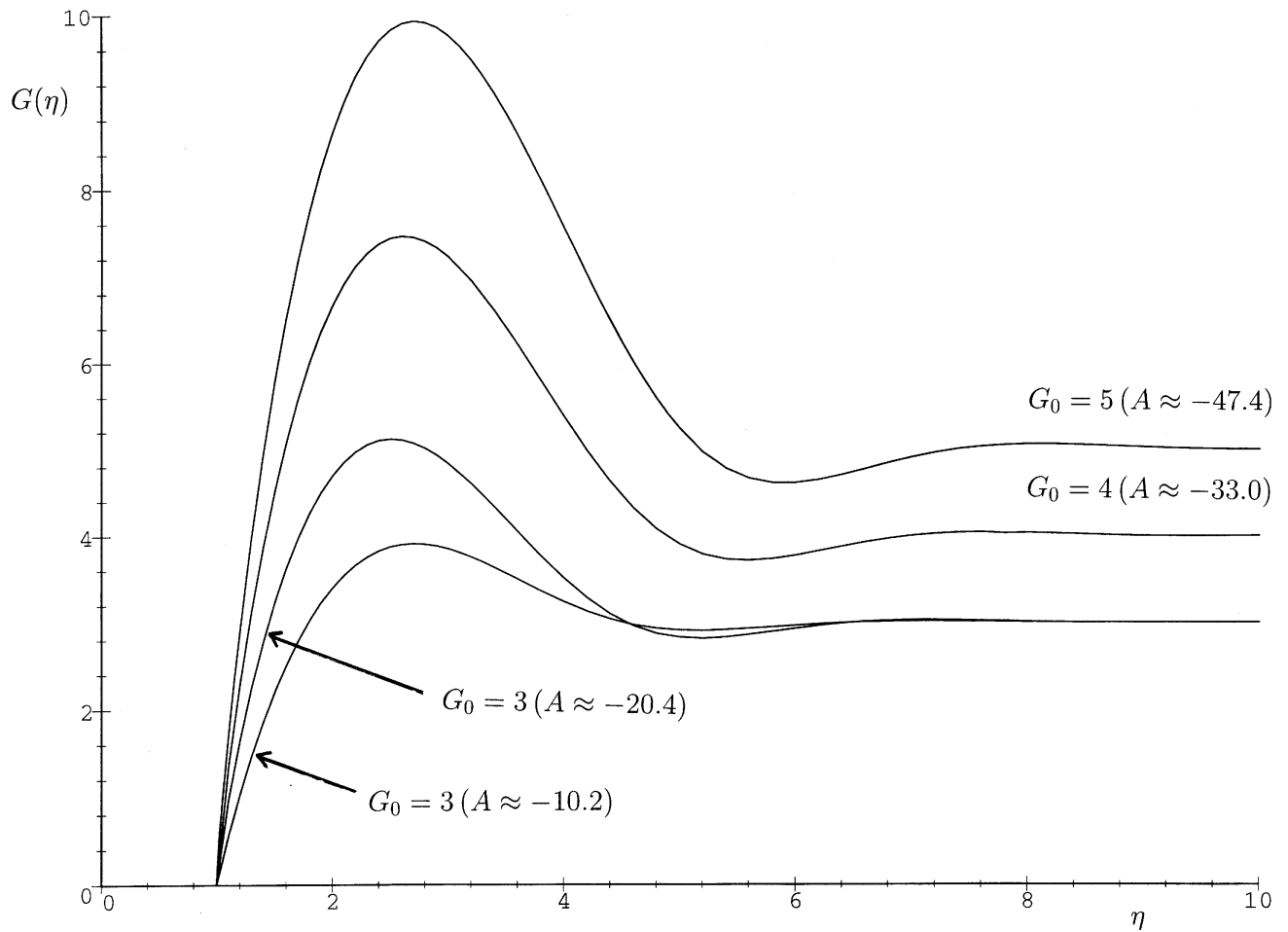

FIGURE 7. Numerically calculated free-surface profiles $G(\eta)$ in case 2 plotted as a function of $\eta$ when $\phi=0$ for $G_{0}=3$ (two solutions with $A \approx-10.2$ and $A \approx-20.4$, respectively), $G_{0}=4$ (one solution with $A \approx-33.0$ ) and $G_{0}=5$ (one solution with $A \approx-47.4$ ).

$G_{0}<G_{0 c}$, where $G_{0 c} \approx 2.4$, but for $G_{0} \geqslant G_{0 c}$ there is a one-parameter family of solutions for $G$ for a range of values of $\phi$ in equation (5.7) whose extent depends on $G_{0}$.

In practice, we would expect to prescribe either the average volume flux per unit width, $Q$, or equivalently the uniform height of the layer far from the dry patch, $b G_{0}$. In either case the parameter $c$ remains undetermined and so each of the solutions described above corresponds to a one-parameter family of possible solutions. This behaviour is similar to that of the corresponding rivulet solutions obtained by Smith [25] and Duffy \& Moffatt [6], in which specifying the value of the volume flux, $Q_{\mathrm{R}}$, determines the value of $c$ uniquely in the former case, but yields a one-parameter family of possible solutions in the latter case.

Both solutions described above are valid only provided that $|n y| \ll|x|$ and away from the contact line where the lubrication approximation fails because they have infinite slope. The corresponding rivulet similarity solutions of Smith [25] and Duffy \& Moffatt [6] discussed in $\S 3$ are also not valid near the contact line, but for a different reason, namely that they have non-constant (but finite) slope there. In the appendix we show how these rivulet similarity solutions can be modified to accommodate a non-self-similar fixed-contact-angle condition at the contact line by incorporating sufficiently strong slip into the model provided that the prescribed contact angle is sufficiently small for the lubrication approximation to remain valid. Unfortunately, this procedure does not work 


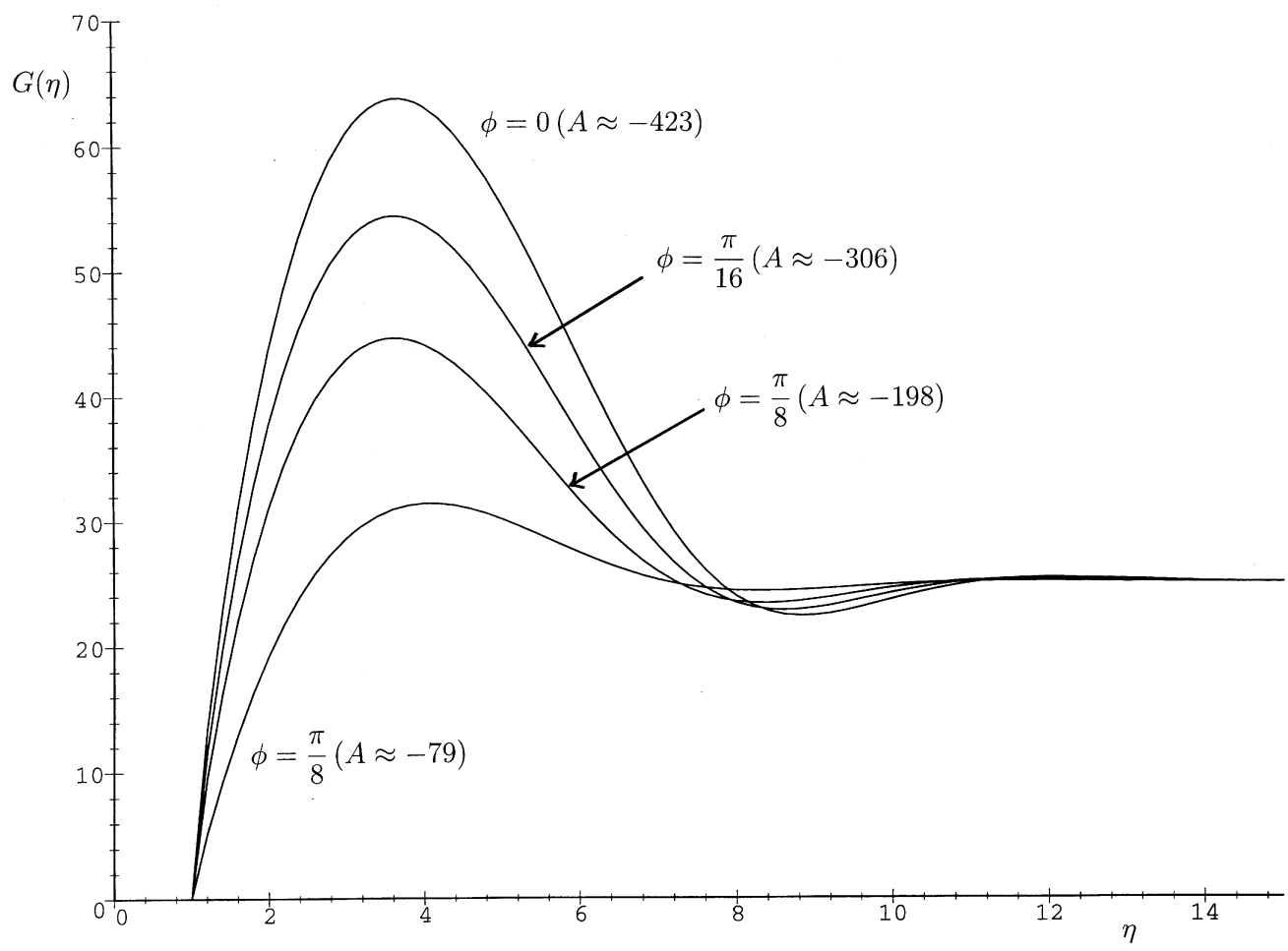

FigURE 8 . Numerically calculated free-surface profiles $G(\eta)$ in case 2 plotted as a function of $\eta$ when $G_{0}=25$ for $\phi=0$ (one solution with $A \approx-423$ ), $\phi=\pi / 16$ (one solution with $A \approx-306$ ) and $\phi=\pi / 8$ (two solutions with $A \approx-79$ and $A \approx-198$, respectively).

for the present dry-patch similarity solutions; presumably in this case the lubrication approximation must be abandoned and it is necessary to formulate and solve appropriate Stokes flow problems near the contact line and then match these solutions with the relevant similarity solutions far from the contact line for all values of the prescribed constant contact angle.

A quantity of some interest which can be calculated from the present solutions is the difference between the volume flux of a liquid in a uniform film and that of a film containing a dry patch with the same uniform height far from the patch. This difference in flux, denoted by $\Delta Q$, is given by

$$
\Delta Q=\frac{2 \rho g \sin \alpha}{3 \mu} f^{3} y_{\mathrm{e}}\left[\lim _{\eta \rightarrow \infty} \int_{1}^{\eta} G(\hat{\eta})^{3}-G_{0}^{3} \mathrm{~d} \hat{\eta}-G_{0}^{3}\right] .
$$

Straightforward integration by parts employing the appropriate governing equations and asymptotic solutions as $\eta \downarrow 1$ and $\eta \rightarrow \infty$ yields

$$
\Delta Q=-\frac{\rho g \sin \alpha}{6 \mu}\left(\frac{c \tan \alpha}{2}\right)^{3} a^{4}(c x)^{\frac{1}{2}}
$$

in case 1 and

$$
\Delta Q=\frac{5 \rho g \sin \alpha}{32 \mu}\left(\frac{c \rho g \sin \alpha}{4 \sigma}\right)^{3} a^{4}(c x)^{\frac{1}{4}}
$$


in case 2 , i.e. the presence of the dry patch always decreases the flux relative to the uniform film in case 1 but always increases it in case 2 .

While there is insufficient available experimental data to permit a quantitative comparison, the solutions presented here evidently capture some of the qualitative features observed in experiments. In particular, the solution in case 2 predicts the presence of the capillary ridge near the apex of the dry patch described by Murgatroyd [17] and Podgorski et al. $[19,20]$. However, like the approximate model of Podgorski et al. $[19,20]$, the present solution is not able to predict the maximum critical volume flux observed experimentally for a dry patch to occur without invoking an additional assumption. Indeed, the present work shows that, while solutions are possible for all values of the volume flux in case 1, a minimum volume flux is required for solutions to exist in case 2 .

Finally, we note that while the present solutions for $0<\alpha<\pi / 2$ represent flow around a widening dry patch on the upper surface of an inclined plane, the corresponding results when $\pi / 2<\alpha<\pi$ represent flow around a dry patch on the lower surface of an inclined plane. However, whereas the present solution in case 2 applies immediately to the latter situation (and indeed also applies in the special case of a vertical plane $\alpha=\pi / 2$ ), the present solution in case 1 makes physical sense in the latter situation only if $c<0$ and $x<0$, and hence it represents flow around a narrowing dry patch. The analogous interpretation of Smith's [25] rivulet solution was noted by Duffy \& Moffatt [6].

\section{Acknowledgements}

This work was begun while the first author (SKW) was a Visiting Scholar in the Department of Engineering Sciences and Applied Mathematics of Northwestern University where he was partially supported under a United States Department of Energy Grant in the Basic Energy Sciences while he was visiting Professor S. G. Bankoff and Professor S. H. Davis. The first author gratefully acknowledges valuable discussions with Professor S. G. Bankoff and Professor M. J. Miksis (Northwestern University) and Professor J. R. King (University of Nottingham) during the course of the present work.

\section{Appendix: Imposing a fixed-contact-angle condition at the contact line of the rivulet similarity solutions of Smith [25] and Duffy \& Moffatt [6]}

In this appendix we address the question of imposing a fixed-contact-angle condition at the contact line of the rivulet similarity solutions of Smith [25] and Duffy \& Moffatt [6]. Evidently the form of these similarity solutions in which $h_{y}=b(c x)^{m-n} G^{\prime}(\eta)$ is, in general, incompatible with a fixed-contact-angle condition. Specifically, the 'contact angle' varies with $x$ like $x^{-4 / 7}$ and $x^{-4 / 13}$, respectively. It is therefore reasonable to ask if we can modify these solutions in an 'inner' region (or regions) near the contact line in order to accommodate the non-self-similar fixed-contact-angle condition $h_{y}=-\theta$ at $\eta=1$, where $\theta$ is a prescribed constant contact angle.

To impose a fixed-contact-angle condition at the contact line we replace the no-slip boundary conditions at the substrate, $u=v=0$ at $y=0$, by the slip conditions $u=\beta u_{z} / 3$ and $v=\beta v_{z} / 3$ at $y=0$, where $\beta=\beta(h)$ is the (small) slip length. Conceptually similar approaches have been used by Silliman \& Scriven [24] (who introduced slip in order to 
alleviate the shear-stress singularity that would otherwise occur in steady viscous flow at a channel exit), and by Hocking [10] (who employed a specific slip model to complete Huppert's [11] unsteady similarity solution describing an elongating thin liquid sheet). With this new slip condition equation (2.2) becomes

$$
\left[h^{2}(h+\beta)\left(\rho g h \cos \alpha-\sigma h_{y y}\right)_{y}\right]_{y}-\rho g \sin \alpha\left[h^{2}(h+\beta)\right]_{x}=0 .
$$

Several different models have been proposed for $\beta$; here we consider the fairly general form $\beta=\epsilon^{N+1} / h^{N}$, where $\epsilon$ is the small constant slip length and the exponent $N \geqslant 0$. In the cases $N=0$ and $N=1$ we recover the familiar models $\beta=\epsilon$ and $\beta=\epsilon^{2} / h$ first proposed by Navier (see, for example, Hocking [9]) and Greenspan [7], respectively. We anticipate that slip will be significant in a small region of size $O(\epsilon)$ near the contact line in which $h_{y}=O(1)$, and so we introduce appropriately rescaled local variables defined by $y=(c x)^{n}-\epsilon Y$ and $h=\epsilon H$, where $H=H(x, Y)$. At leading order in $\epsilon$, equation (A 1) becomes simply

$$
H^{2}\left(H+H^{-N}\right) H_{Y Y Y}=K,
$$

where $K=K(x)$ is an unknown function of $x$ only. In the limit $H \downarrow 0$ equation (A 2) becomes $H_{Y Y Y}=K H^{N-2}$ and so will permit a solution in the form $H=\theta Y+o(Y)$ as $Y \downarrow 0$ only if $N>0$, i.e. provided that the slip is sufficiently strong. Specifically, as $Y \downarrow 0$ we have $H=\theta Y+O\left(Y^{N+1}\right)$ when $0<N<1, H=\theta Y+O\left(Y^{2} \log Y\right)$ when $N=1$, and $H=\theta Y+O\left(Y^{2}\right)$ when $N>1$. In the limit $H \rightarrow \infty$ equation (A 2) becomes $H_{Y Y Y}=K H^{-3}$, which has an asymptotic solution

$$
H \sim A Y+\frac{K}{2 A^{3}} \log Y
$$

as $Y \rightarrow \infty$, where $A=A(x)$ is an unknown function of $x$ only.

In the case of strong surface tension investigated by Duffy \& Moffatt [6] (corresponding to the present case 2), the outer limit of the inner solution given by equation (A 3) matches directly to the inner limit of the outer solution, namely

$$
h \sim \frac{3 c \rho g \sin \alpha}{13 \sigma}\left(\frac{24 \lambda-1}{12}\right)\left(1-\frac{y}{(c x)^{\frac{3}{13}}}\right)(c x)^{-\frac{1}{13}}
$$

as $y \uparrow(c x)^{3 / 13}$, provided that

$$
A=\frac{3 \operatorname{cog} \sin \alpha}{13 \sigma}\left(\frac{24 \lambda-1}{12}\right)(c x)^{-\frac{4}{13}} .
$$

This analysis is for $\lambda>1 / 24$; in the special case $\lambda=1 / 24$ the similarity solution has zero slope at the contact line for all values of $x$.

In the case of weak surface tension investigated by Smith [25] (corresponding to the present case 1), the situation is somewhat more complicated. Inspection of equation (A 1) shows that however weak surface-tension effects are, they will be significant in a small region of size $O(\delta)$ near the contact line, where $\delta=(\sigma / \rho g \cos \alpha)^{1 / 2}$. Assuming that $\epsilon \ll \delta$ the outer limit of the inner solution given by equation (A 3) matches the inner limit of the outer solution, namely

$$
h \sim \frac{3 c \tan \alpha}{7}\left(1-\frac{y}{(c x)^{\frac{3}{7}}}\right)(c x)^{-\frac{1}{7}}
$$


as $y \uparrow(c x)^{3 / 7}$, via an 'intermediate' region in which $y=(c x)^{3 / 7}-\delta \hat{Y}$ and $h=\delta \hat{H}$, where $\hat{H}=\hat{H}(x, \hat{Y})$. In this intermediate region the leading-order version of equation (A 1) is

$$
\hat{H}^{3}\left[\left(\hat{H}-\hat{H}_{\hat{Y} \hat{Y}}\right)_{\hat{Y}}-\frac{3 c \tan \alpha}{7}(c x)^{-\frac{4}{7}}\right]=-K,
$$

whose solutions match with equation (A 3) as $\hat{Y} \downarrow 0$ and equation (A 6) as $\hat{Y} \rightarrow \infty$.

In conclusion, it appears that we can indeed always modify the rivulet similarity solutions of Smith [25] and Duffy \& Moffatt [6] to accommodate a non-self-similar fixedcontact-angle condition at the contact line by incorporating sufficiently strong slip into the model. Evidently the procedure described above is appropriate only if the prescribed contact angle $\theta$ is sufficiently small for the lubrication approximation to remain valid in the inner and intermediate regions. If $\theta$ is too large then presumably the corresponding (but more complicated) Stokes flow problems would have to be formulated and solved in these regions.

Note that this procedure does not work for the present dry-patch problems. For example, in case 2 the corresponding analysis leads to the requirement that $a=O(\epsilon)$, which is inconsistent with the a priori assumption that $a=O(1)$. Presumably in this case the lubrication approximation must be abandoned and it is necessary to formulate and solve appropriate Stokes flow problems near the contact line and then match these solutions with the relevant similarity solutions far from the contact line for all values of the prescribed constant contact angle.

\section{References}

[1] Allen, R. F. \& Biggin, C. M. (1974) Longitudinal flow of a lenticular liquid filament down an inclined plane. Physics of Fluids, 17, 287-291.

[2] Bankoff, S. G. (1971) Minimum thickness of a draining liquid film. Int. J. Heat \& Mass Transfer, 14, 2143-2146.

[3] Chung, J. C. \& Bankoff, S. G. (1980) Initial breakdown of a heated liquid film in cocurrent two-component annular flow: II. Rivulet and drypatch models. Chemical Eng. Comm. 4, 455-470.

[4] Davis, S. H. (1980) Moving contact lines and rivulet instabilities. Part 1. The static rivulet. J. Fluid Mechanics, 98, 225-242.

[5] Duffy, B. R. \& Moffatt, H. K. (1995) Flow of a viscous trickle on a slowly varying incline. Chemical Eng. J. 60, 141-146.

[6] Duffy, B. R. \& Moffatt, H. K. (1997) A similarity solution for viscous source flow on a vertical plane. Euro. J. Appl. Math. 8, 37-47.

[7] Greenspan, H. P. (1978) On the motion of a small viscous droplet that wets a surface. J. Fluid Mech. 84, 125-143.

[8] Hartley, D. E. \& Murgatroyd, W. (1964) Criteria for the break-up of thin liquid layers flowing isothermally over solid surfaces. Int. J. Heat \& Mass Transfer, 7, 1003-1015.

[9] Hocking, L. M. (1981) Sliding and spreading of thin two-dimensional drops. Quart. J. Mech. \& Appl. Math. 34, 37-55.

[10] Hocking, L. M. (1990) Spreading and instability of a viscous fluid sheet. J. Fluid Mech. 211, 373-392.

[11] Huppert, H. E. (1982) Flow and instability of a viscous current down a slope. Nature, 300, 427-429.

[12] López, P. G., Miksis, M. J. \& Bankoff, S. G. (1997) Stability and evolution of a dry spot. Northwestern University Applied Mathematics Technical Report 9605. 
[13] McPherson, G. D. (1970) Axial stability of the dry patch formed in dryout of a two-phase annular flow. Int. J. Heat \& Mass Transfer, 13, 1133-1152.

[14] Mikielewicz, J. \& Moszynski, J. R. (1976) Minimum thickness of a liquid film flowing vertically down a solid surface. Int. J. Heat \& Mass Transfer, 19, 771-776.

[15] Mikielewicz, J. \& Moszynski, J. R. (1978) An improved analysis of breakdown of thin liquid films. Arch. Mech. 30, 489-500.

[16] Moriarty, J. A. \& Schwartz, L. W. (1993) Dynamic considerations in the closing and opening of holes in thin liquid films. J. Colloid \& Interface Sci. 161, 335-342.

[17] Murgatroyd, W. (1965) The role of shear and form forces in the stability of a dry patch in two-phase film flow. Int. J. Heat \& Mass Transfer, 8, 297-301.

[18] Oron, A., Davis, S. H. \& Bankoff, S. G. (1997) Long-scale evolution of thin liquid films. Rev. Mod. Phys. 69, 931-980.

[19] Podgorski, T., Limat, L., Flesselles, J.-M., Callegari, G. \& Jenffer, P. (1998) Dewetting of flowing films. Proc. 2nd Euro. Coating Symposium (Euromech 367), pp. 450-459. Strasbourg, France.

[20] Podgorski, T., Flesselles, J.-M. \& Limat, L. (1999) Dry arches within flowing films. Phys. Fluids, 11, 845-852.

[21] Ponter, A. B., Davies, G. A., Ross, T. K. \& Thornley, P. G. (1967) The influence of mass transfer on liquid film breakdown. Int. J. Heat \& Mass Transfer, 10, 349-359.

[22] Schmuki, P. \& Laso, M. (1990) On the stability of rivulet flow. J. Fluid Mech. 215, 125-143.

[23] Sharma, A. \& Ruckenstein, E. (1989) Dewetting of solids by the formation of holes in macroscopic liquid films. J. Colloid \& Interface Sci. 133, 358-368.

[24] Silliman, W. J. \& Scriven, L. E. (1978) Slip of liquid inside a channel exit. Phys. Fluids, 21, 2115-2116.

[25] Sмiтh, P. C. (1973) A similarity solution for slow viscous flow down an inclined plane. J. Fluid Mech. 58, 275-288.

[26] Taylor, G. I. \& Michael, D. H. (1973) On making holes in a sheet of fluid. J. Fluid Mech. 58, 625-639.

[27] Towell, G. D. \& Rothfeld, L. B. (1966) Hydrodynamics of rivulet flow. Am. Inst. Chem. Eng. J. 12, 972-980.

[28] Troian, S. M., Herbolzheimer, E., Safran, S. A. \& Joanny, J. F. (1989) Fingering instabilities of driven spreading films. Europhys. Lett. 10, 25-30.

[29] Weiland, R. H. \& Davis, S. H. (1981) Moving contact lines and rivulet instabilities. Part 2. Long waves on flat rivulets. J. Fluid Mech. 107, 261-280.

[30] Wilson, S. D. R. (1974) The stability of a dry patch on a wetted wall. Int. J. Heat \& Mass Transfer, 17, 1607-1615.

[31] Wilson, S. K. \& Duffy, B. R. (1996) An asymptotic analysis of small holes in thin fluid layers. J. Eng. Math. 30, 445-457.

[32] Wilson, S. K. \& Duffy, B. R. (1998) On the gravity-driven draining of a rivulet of viscous fluid down a slowly varying substrate with variation transverse to the direction of flow. Phys. Fluids 10, 13-22.

[33] Wilson, S. K. \& Terrill, E. L. (1996) The dynamics of planar and axisymmetric holes in thin fluid layers. 1st Euro. Coating Symposium on the Mechanics of Thin Film Coatings, pp. 288-297. Leeds, UK. World Scientific.

[34] Young, G. W. \& Davis, S. H. (1987) Rivulet instabilities. J. Fluid Mech. 176, 1-31.

[35] Zuber, N. \& Staub, F. W. (1966) Stability of dry patches forming in liquid films flowing over heated surfaces. Int. J. Heat \& Mass Transfer, 9, 897-905. 